\title{
Multipole modes of excitation in triaxially deformed superfluid nuclei
}

\author{
Kouhei Washiyama ${ }^{1, *}$ and Takashi Nakatsukasa ${ }^{1,2,3}$ \\ ${ }^{1}$ Center for Computational Sciences, University of Tsukuba, Tsukuba 305-8577, Japan \\ ${ }^{2}$ Faculty of Pure and Applied Sciences, University of Tsukuba, Tsukuba 305-8571, Japan \\ ${ }^{3}$ iTHES, RIKEN, Wako 351-0198, Japan
}

(Received 22 August 2017; published 30 October 2017)

\begin{abstract}
Background: The five-dimensional quadrupole collective model based on energy density functionals (EDFs) has often been employed to treat long-range correlations associated with shape fluctuations in nuclei. Our goal is to derive the collective inertial functions in the collective Hamiltonian by the local quasiparticle random-phase approximation (QRPA) that correctly takes into account time-odd mean-field effects. Currently, a practical framework to perform the QRPA calculation with the modern EDFs on the $(\beta, \gamma)$ deformation space is not available.

Purpose: Toward this goal, we develop an efficient numerical method to perform the QRPA calculation on the $(\beta, \gamma)$ deformation space based on the Skyrme EDF.

Methods: We use the finite amplitude method (FAM) for the efficient calculation of QRPA strength functions for multipole external fields. We construct a computational code of FAM-QRPA in the three-dimensional Cartesian coordinate space to handle triaxially deformed superfluid nuclei.

Results: We validate our new code by comparing our results with former QRPA calculations for axially symmetric nuclei. Isoscalar quadrupole strength functions in triaxial superfluid nuclei ${ }^{110} \mathrm{Ru}$ and ${ }^{190} \mathrm{Pt}$ are obtained within a reasonable computational cost.

Conclusions: QRPA calculations for triaxially deformed superfluid nuclei based on the Skyrme EDF are achieved with the help of the FAM. This is an important step toward the microscopic calculation of collective inertial functions of the local QRPA.
\end{abstract}

DOI: 10.1103/PhysRevC.96.041304

Introduction. The shape of atomic nuclei is influenced strongly by the quantum nature of nuclear systems. Excitation spectra and their transition probabilities clearly indicate the existence of shape fluctuations and shape coexistence [1], particularly in transitional regions from spherical to deformed shapes in the ground state. The long-lived fission products (LLFP) from uranium fueled reactors, such as $\mathrm{Pd}$ and $\mathrm{Zr}$ isotopes, are located in transitional regions on the nuclear chart and demonstrate the shape mixing and coexistence. It is important to understand the basic properties of the LLFPs to develop a possible nuclear transmutation method, which is the main target of an ImPACT program "Reduction and Resource Recycling of High-level Radioactive Wastes through Nuclear Transmutation" [2].

One of the standard methods of investigating nuclear manybody problems is the nuclear energy density functional (EDF) theory [3]. The nuclear EDF well describes the ground-state properties of atomic nuclei. However, on the mean-field level, it cannot describe shape fluctuations and shape coexistence. We need to go beyond the mean field for a description of such phenomena, including quantum fluctuations associated with the large-amplitude collective motion. If the EDF were constructed as an expectation value of a well-defined Hamiltonian, a possible extension would be the generator coordinate method (GCM) [4-6]. However, most of the EDFs are known to have a singular behavior $[7,8]$, which prevents us from the straightforward application of the GCM.

\footnotetext{
*washiyama@nucl.ph.tsukuba.ac.jp
}

A practical alternative to the GCM may be the collective Hamiltonian method. The five-dimensional quadrupole collective Hamiltonian with quadrupole deformation parameters $(\beta, \gamma)$ and three Euler angles is constructed from the EDFbased calculation of Skyrme, Gogny, and covariant EDFs [9-11]. The collective potential is obtained by the constrained minimization of the EDF, whereas for the collective inertial functions, the Inglis-Belyaev cranking formula is employed. Therefore, the time-odd components in the mean field are neglected in those studies, and an empirical enhancement factor of 1.2-1.4 is often adopted for the collective inertias.

Starting from the adiabatic self-consistent collective coordinate method [12-14], Hinohara et al. microscopically constructed the quadrupole collective Hamiltonian [15]. The collective potential is provided by the Hartree-FockBogoliubov (HFB) calculation with constraints on the $(\beta, \gamma)$ values. The collective inertial functions are given as those of normal modes of the local quasiparticle random-phase approximation (QRPA), which properly includes time-odd components in the mean field. The numerical calculation was performed with the pairing-plus-quadrupole $(\mathrm{P}+\mathrm{Q})$ Hamiltonian. They showed a significant time-odd effect on collective inertial functions and on excitation levels in nuclei.

Our goal is an extension of the work by Hinohara and coworkers [15-17], that is, to replace the semiphenomenological $\mathrm{P}+\mathrm{Q}$ Hamiltonian by modern Skyrme EDFs. It will also be an extension to include the $\gamma$ degree of freedom from the work of Yoshida and Hinohara [18] that constructed a threedimensional (3D) quadrupole collective Hamiltonian based on the Skyrme EDF restricted to axially symmetric shapes. 
In order to achieve this goal, the challenge is to perform the local QRPA with a Skyrme EDF at the constrained HFB states of triaxial shapes. The axially deformed QRPA calculations with the modern Skyrme, Gogny, and covariant EDFs have recently become available [19-23], and the 3D RPA calculations without pairing were achieved [24-27]. However, currently, an efficient framework to solve the self-consistent QRPA with modern EDFs applicable to triaxial shapes is still missing, although there are some related studies using the real-time method [28-32].

In this Rapid Communication, as a first step toward the goal, we construct an efficient QRPA solver for triaxially deformed superfluid nuclei with the Skyrme EDF with the help of the finite amplitude method (FAM) [33,34] (see Refs. [25,26,35-51] for applications of the FAM). We start from the HFB code using the two-basis method of the 3D Cartesian coordinate representation. We apply the method to multipole modes of excitation in triaxial nuclei as well as in axially symmetric nuclei for a benchmark and show its feasibility.

Development of the $3 D$ FAM-QRPA. Since the details of the derivation of the FAM equations for the QRPA can be found in Refs. [34,35], here we recapitulate the basic idea and formulas of the FAM. We start from the linear-response equation,

$$
\begin{aligned}
\left(E_{\mu}+E_{\nu}-\omega\right) X_{\mu \nu}(\omega)+\delta H_{\mu \nu}^{20}(\omega) & =-F_{\mu \nu}^{20}, \\
\left(E_{\mu}+E_{\nu}+\omega\right) Y_{\mu \nu}(\omega)+\delta H_{\mu \nu}^{02}(\omega) & =-F_{\mu \nu}^{02},
\end{aligned}
$$

where $X$ and $Y$ are FAM amplitudes at a given frequency $\omega . \delta H^{20(02)}$ and $F^{20(02)}$ are two-quasiparticle matrix elements of an induced Hamiltonian and an external field, respectively [34].

The FAM equation is solved iteratively at each $\omega$. First, from the $X$ and $Y$ amplitudes at the previous iteration, the induced density $\delta \rho$ and pairing tensors $\delta \kappa$ and $\overline{\delta \kappa}$ are calculated as

$$
\begin{aligned}
& \delta \rho=U X V^{T}+V^{*} Y^{T} U^{\dagger}, \\
& \delta \kappa=U X U^{T}+V^{*} Y^{T} V^{\dagger}, \\
& \overline{\delta \kappa}=V^{*} X^{\dagger} V^{\dagger}+U Y^{*} U^{T},
\end{aligned}
$$

where $U$ and $V$ matrices are taken from the HFB ground state. The induced pair density has two independent components; $\delta \kappa$ is proportional to $e^{-i \omega t}$ and the other $\overline{\delta \kappa}$ is proportional to $e^{i \omega t}$ [34].

Next, induced HF Hamiltonian $\delta h$ and pair fields $\delta \Delta$ and $\overline{\delta \Delta}$ are obtained using a small real parameter $\eta$ as

$$
\begin{aligned}
\delta h & =\frac{1}{\eta}(h[\rho+\eta \delta \rho]-h[\rho])=h^{(1)}[\delta \rho]+\delta h^{(\alpha)}[\rho, \delta \rho], \\
\delta \Delta & =\frac{1}{\eta}(\Delta[\kappa+\eta \delta \kappa]-\Delta[\kappa])=\Delta[\delta \kappa], \\
\overline{\delta \Delta} & =\frac{1}{\eta}(\Delta[\kappa+\eta \overline{\delta \kappa}]-\Delta[\kappa])=\Delta[\overline{\delta \kappa}],
\end{aligned}
$$

where $\rho$ and $\kappa$ are the density and pair tensor in the ground state, respectively. Most of the terms in the HF Hamiltonian $h$ linearly depend on $\rho$, whereas there is a term with density dependence of fractional power $\rho^{\alpha}$. We denote here the former as $h^{(1)}$ and the latter as $h^{(\alpha)} ; h=h^{(1)}+h^{(\alpha)}$. At the last equality for each field in Eq. (5), the explicit linearization with respect to induced densities is performed for $\delta h^{(\alpha)}$, whereas the rest of the terms can be obtained by simply replacing $\rho$ by $\delta \rho$. In this Rapid Communication, we use the volume-type pairing without density dependence, thus, $\delta \Delta$ can be calculated as the last equation of Eq. (5b). If the pair field $\Delta$ has a density dependence, the explicit linearization is required for $\Delta$, similar to $\delta h^{(\alpha)}$. In the present scheme [44], the induced fields (5) do not depend on $\eta$, which was required by the original FAM formulation [33].

Finally, $\delta H^{20}$ and $\delta H^{02}$ are constructed from $\delta h, \delta \kappa$, and $\overline{\delta \kappa}$, and then, new $X$ and $Y$ amplitudes are obtained from Eq. (1). We employ the modified Broyden method [52] for the FAM iterations. The convergence is reached in about 60-70 iterations at most when the convergence condition is set as the maximum difference between two successive iterations of $X$ and $Y$ less than $10^{-7} ;\left|X_{\mu \nu}^{(n)}-X_{\mu \nu}^{(n-1)}\right|<10^{-7}$ and $\left|Y_{\mu \nu}^{(n)}-Y_{\mu \nu}^{(n-1)}\right|<10^{-7}$ for $\forall \mu \nu$. The imaginary part of the frequency $\omega$ has been introduced as $\omega \rightarrow \omega+i \gamma$ with $\gamma=0.5 \mathrm{MeV}$. The spacing in discretized $\omega$ is taken to be $0.25 \mathrm{MeV}$ to compute strength functions in the following.

The FAM strength function at each $\omega$ is obtained with the converged $X(\omega)$ and $Y(\omega)$ amplitudes as

$$
S(\omega)=-\frac{1}{\pi} \operatorname{Im}\left(\sum_{\mu<\nu} F_{\mu \nu}^{20 *} X_{\mu \nu}(\omega)+F_{\mu \nu}^{02 *} Y_{\mu \nu}(\omega)\right) .
$$

We use the one-body external operators as $\sum_{i=1}^{A} e_{i}^{\text {eff }} f_{L K}\left(\boldsymbol{r}_{i}\right)$ with $f_{L K}\left(\boldsymbol{r}_{i}\right)=r_{i}^{L} Y_{L K}\left(\hat{\boldsymbol{r}}_{i}\right)$ and with $f_{00}\left(\boldsymbol{r}_{i}\right)=r_{i}^{2}$ for the monopole operator. The effective charge is adopted as $e^{\text {eff }}=$ $e Z / A$ for the isoscalar operators and for isovector operators $e^{\mathrm{eff}}=e Z / A(-e N / A)$ for neutrons (protons). We define the quadrupole operators with the $x$-signature quantum number of $r_{x}= \pm 1$ as $Q_{2 K}^{( \pm)}=\left(f_{2 K} \pm f_{2-K}\right) / \sqrt{2}$ for $K>0$. These operators are written in a simple form in terms of the Cartesian coordinate $(x, y, z)$ and convenient in the 3D code. Choosing the $z$ axis as the symmetry axis, the strength function for $Q_{2 K}^{(+)}$ in axially symmetric nuclei is identical to that for $Q_{2 K}^{(-)}$. For spherical nuclei, all the quadrupole operators with different $K$ and $r_{x}$ quantum numbers carry equal strengths.

We have constructed a 3D FAM-QRPA code based on the 3D Skyrme-HFB code CR8 [53-55], which is an extension of the 3D HF + BCS code EV8 [56,57]. The ground state is obtained by the two-basis method $[54,55]$ where the HF basis that diagonalizes the HF Hamiltonian and the canonical basis that diagonalizes the density matrix are used simultaneously. The single-particle wave functions are represented on the square mesh in the 3D Cartesian space and eigenstates of the $z$ signature, the parity, and the $y$ time simplex. As a result, each single-particle wave function has a specific reflection symmetry about the $x=0, y=0$, and $z=0$ planes [53,56-59]. We take into account these symmetry properties when calculating two-quasiparticle matrix elements of the induced densities and fields in the FAM equations, which significantly reduce the computational task. The working volume is then limited to only $1 / 8$ space $(x>0, y>0, z>0)$ of the whole volume for both HFB and FAM computations. 
The mesh spacing of $\Delta x=\Delta y=\Delta z=0.8 \mathrm{fm}$ is used in HFB and the FAM. Note that, since the present FAM code can calculate only excitation modes which conserve parity and $z$-signature symmetries, the $K=1$ modes of quadrupole excitation $\left(Q_{21}^{( \pm)} \sim y z\right.$ and $\left.z x\right)$, which violate the $z$-signature symmetry, cannot be computed. For this case, we rotate the ground-state wave functions to switch the labeling of the axes $(x, y, z)$ so as to perform $y z(z x) \rightarrow x y$. Then, these modes conserve the $z$ signature, and the present FAM code can handle these.

We used SkM* [60] and SLy4 [61] parametrizations, which have been used widely and known to be stable for the QRPA calculations. We used the volume pairing with a pairing window of $20 \mathrm{MeV}$ above and below the Fermi energy in the HF basis described in Refs. [56-58]. We applied the same pairing-cutoff procedure for both HFB and FAM-QRPA calculations. The pairing strength was determined so as to reproduce the neutron pairing gap of $1.25 \mathrm{MeV}$ in ${ }^{120} \mathrm{Sn}$. For simplicity, we used the same pairing strength for neutrons and protons.

Before showing our results, we note the treatment of the boundary condition in the HFB calculations. The continuum (positive-energy) HF states in the cubic boundary condition, which has been used in the codes CR8 and EV8, violate the spherical symmetry in our FAM calculation. For the isoscalar quadrupole modes in the spherical nucleus ${ }^{20} \mathrm{O}$, the strengths at energies around the giant resonance vary depending on $K$ (about a 30\% difference at most). To avoid this symmetryviolation effect, we try to mimic the sphere-type boundary condition, namely, add artificial potentials to the HF potential $V_{\mathrm{sph}}(r)=1000 \mathrm{MeV}$ at $r>R_{\max }$ and $V_{\mathrm{sph}}(r)=0$ at $r<$ $R_{\max }$. We confirmed that this change in the boundary condition does not affect the ground-state property. Furthermore, we obtained that the difference in the giant resonance strengths among different $K$ modes is at most $4 \%$ for ${ }^{20} \mathrm{O}$, which is the same order of the deviation observed in the unperturbed strengths of different $K$ modes.

Results. We first compute isoscalar quadrupole modes of an axially symmetric nucleus ${ }^{24} \mathrm{Mg}$ with the $\mathrm{SkM}^{*} \mathrm{EDF}$ to test our computational code. We adopt the square mesh space of $15^{3}$ and $R_{\max }=12.4 \mathrm{fm}$. The number of HF-basis states is 910 for both protons and neutrons. We obtained the prolately deformed ground state with $\beta=0.49$. In this configuration, the pairing vanishes for both neutrons and protons. Figure 1(a) shows the isoscalar quadrupole strengths of ${ }^{24} \mathrm{Mg}$. By comparing our result to a previous FAM investigation based on the axially symmetric HFBTHO in Ref. [44], included in Fig. 1(b), we found good agreement of the peak energies as well as the shapes of the strength functions in each $K$. The widths of the giant resonances for all $K$ modes in our strengths are wider than those in Ref. [44]. The peak of the $K=1$ spurious mode associated with the rotational-symmetry breaking in the ground state appears at a finite energy $(\omega \approx 1.5 \mathrm{MeV})$. This deviation from zero energy is due to the use of the finite mesh size, which was discussed extensively in Ref. [23]. The energy-weighted sum-rule (EWSR) values summed up to $\omega=50 \mathrm{MeV}$ are exhausted by $98.5 \%(K=0)$ and $98.4 \%$ $(K=2)$. The strengths with $r_{x}= \pm 1$ coincide for $K=1$ and 2.

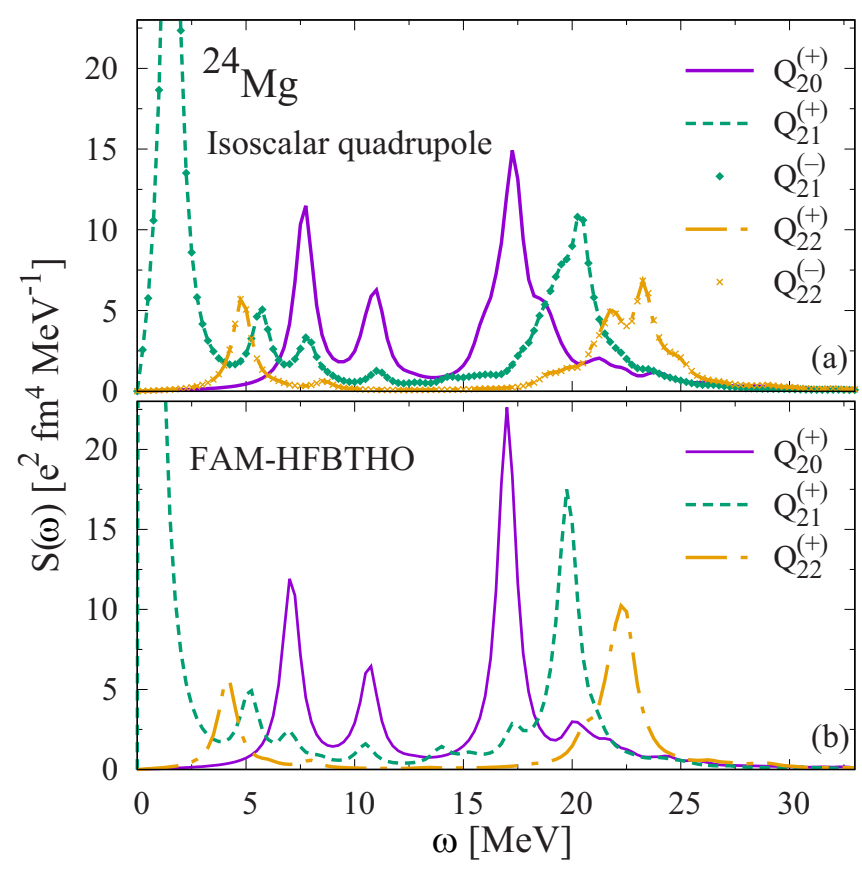

FIG. 1. (a) Isoscalar quadrupole strengths of different $K$ modes as a function of $\omega$ for ${ }^{24} \mathrm{Mg}$ calculated with $\mathrm{SkM}^{*}$ and (b) compared with those in Ref. [44].

Figure 2(a) shows isoscalar and isovector monopole strengths in a prolately deformed superfluid nucleus ${ }^{100} \mathrm{Zr}$ computed with $17^{3} \operatorname{mesh}\left(R_{\max }=14.0 \mathrm{fm}\right)$ and $1120 \mathrm{HF}$-basis states with the SLy4 EDF. The obtained ground state has a finite pairing gap for protons (normal phase in neutrons) and $\beta=0.43$. Compared with the previous axial matrix-form QRPA [63] and FAM-QRPA [35] [included in Fig. 2(b)], nice agreement on the peak energies is obtained, even though we used different pairing functionals and different pairing cutoffs from those in Refs. [35,63]. In Fig. 2(a), we also show the dependence on the pairing window energy. The pairing strength of each pairing window was adjusted with the method mentioned above. No significant dependence of pairing window energy is observed in the strengths. Furthermore, the $0^{+}$spurious modes corresponding to the pair rotation are not seen in the monopole strength function. This indicates good decoupling between the pair and the monopole modes of excitation.

We show the isoscalar quadrupole strengths of ${ }^{92} \mathrm{Zr}$ and ${ }^{94} \mathrm{Zr}$ in Fig. 3, which are next to an LLFP ${ }^{93} \mathrm{Zr}$. The model space was same as in ${ }^{100} \mathrm{Zr}$, but the $\mathrm{SkM}^{*}$ EDF was used. The ground states of ${ }^{92} \mathrm{Zr}$ and ${ }^{94} \mathrm{Zr}$ are spherical and superfluid in both neutrons and protons. Since these nuclei are spherical in their ground states, the strengths of different $K$ modes agree with each other. The giant resonance peaks appear at around $15 \mathrm{MeV}$, whereas we also observe that the lowest peak is located at about $\omega \approx 1 \mathrm{MeV}$. These low-energy modes are expected to play an important role in the shape fluctuation, which will be our future target.

Finally, we show the isoscalar quadrupole modes in triaxially deformed superfluid nuclei. A typical mass region of appearance of the triaxial ground state is the $A \approx 100$ 


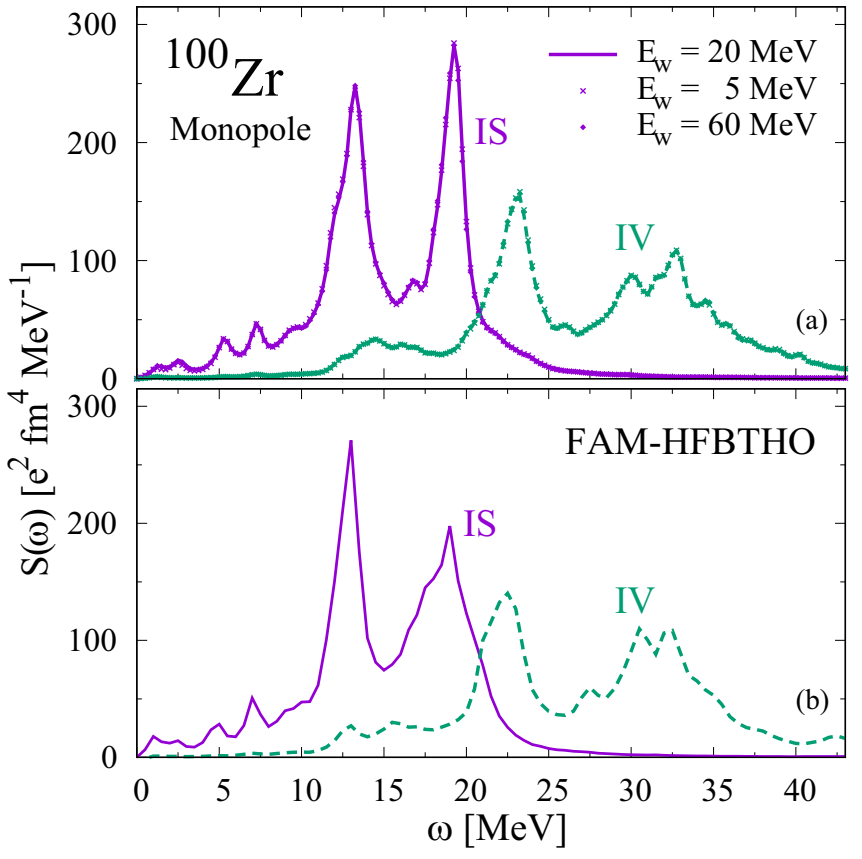

FIG. 2. (a) Isoscalar (IS) and isovector (IV) monopole strengths as a function of $\omega$ for ${ }^{100} \mathrm{Zr}$ with SLy4 and with different pairing window energies $E_{w}=20 \mathrm{MeV}$ (the solid line), $5 \mathrm{MeV}$ (the cross), and $60 \mathrm{MeV}$ (the diamond) and (b) compared with those in Ref. [35] (numerical data from Ref. [62]).

region and $\mathrm{Pt}$ isotopes [31,32]. Here we take ${ }^{110} \mathrm{Ru}$ and ${ }^{190} \mathrm{Pt}$, calculated with the SkM* EDF. We set the longest, middle, and shortest axes to be $z, x$, and $y$ axes, respectively. Note that the magnetic quantum number $K$ is not a good quantum number for triaxial nuclei. For our convenience, however, we use the $K$ values to specify the type of quadrupole operators $Q_{2 K}^{( \pm)}$.

Figures 4(a) and 4(b) show the isoscalar quadrupole strengths of ${ }^{110} \mathrm{Ru}$ and of ${ }^{190} \mathrm{Pt}$, respectively. In both nuclei, neutrons are in the superfluid phase, whereas protons are not. The obtained quadrupole deformation parameters are $\beta=0.32, \gamma=19^{\circ}$ for ${ }^{110} \mathrm{Ru}$ with the same model space as in Fig. 3. For ${ }^{190} \mathrm{Pt}$ with an enlarged model space as $19^{3}$ mesh $\left(R_{\max }=15.6 \mathrm{fm}\right)$ and 1360 HF-basis states, we obtain

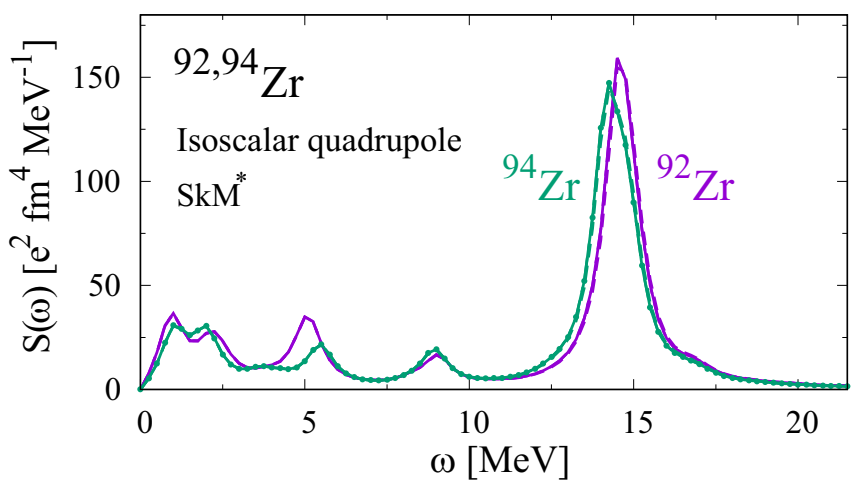

FIG. 3. Isoscalar quadrupole strengths for spherical nuclei ${ }^{92} \mathrm{Zr}$ (purple) and ${ }^{94} \mathrm{Zr}$ (green). All the strengths with $K=0,1$, and 2 (the solid, dashed, and dot-dashed lines) and $r_{x}= \pm 1$ are identical.

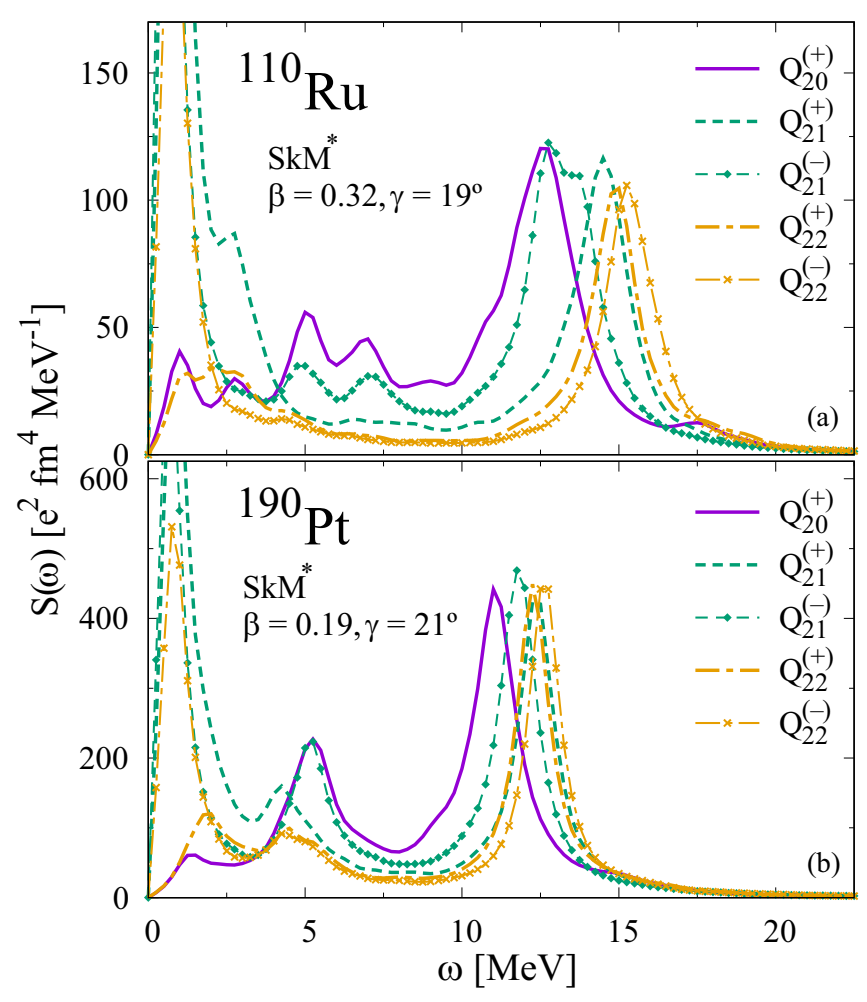

FIG. 4. Isoscalar quadrupole strengths for triaxial nuclei (a) ${ }^{110} \mathrm{Ru}$ and (b) ${ }^{190} \mathrm{Pt}$.

the ground state with $\beta=0.19, \gamma=21^{\circ}$. In both nuclei, our calculation clearly produces additional signature splitting of the strength in which the peaks with different $x$ signatures no longer coincide due to the triaxial deformation. We obtain three spurious modes near zero energy due to the existence of rotations around the $x, y$, and $z$ axes. The EWSR values are well satisfied, $98 \%$ to $99 \%$ for $K=0$ and $\left(K, r_{x}\right)=(2,+)$ modes. We have also confirmed that the isoscalar quadrupole response has no significant dependence on the pairing window in a range from 10 to $50 \mathrm{MeV}$.

For the isoscalar quadrupole $Q_{20}$ of ${ }^{110} \mathrm{Ru}$, we examine in detail the convergence property of the EWSR with respect to the number of HF-basis states. Table I shows the calculated EWSR values with the FAM. For neutrons in $N_{\mathrm{HF}} \leqslant 728$ and

TABLE I. EWSR values summed up to $\omega=50 \mathrm{MeV}$ for the isoscalar quadrupole $K=0$ strength in units of $\mathrm{MeV} e^{2} \mathrm{fm}^{4}$ in ${ }^{110} \mathrm{Ru}$ with different numbers of HF-basis states $N_{\mathrm{HF}}$ and of twoquasiparticle states $N_{2 \mathrm{qp}}$. The last two rows show the maximum quasiparticle energies $E_{\mathrm{OP}}^{\max }$ (in $\mathrm{MeV}$ ) of neutrons (n) and protons (p).

\begin{tabular}{lcccc}
\hline \hline$N_{\mathrm{HF}}$ & $N_{2 \mathrm{qp}}$ & EWSR & $E_{\mathrm{QP}, \mathrm{n}}^{\max }$ & $E_{\mathrm{QP}, \mathrm{p}}^{\max }$ \\
\hline 240 & 7400 & 6912 & 41.3 & 34.2 \\
440 & 24650 & 7121 & 41.3 & 39.8 \\
728 & 67130 & 7160 & 41.3 & 49.5 \\
910 & 104713 & 7164 & 45.9 & 55.3 \\
1120 & 158368 & 7166 & 50.6 & 60.2 \\
\hline \hline
\end{tabular}


protons in $N_{\mathrm{HF}}=240$, the main component of the highest quasiparticle state is the deepest hole state in the HF basis. We reach an approximate convergence of the EWSR at $N_{\mathrm{HF}} \geqslant 728$ which corresponds to the number of two-quasiparticle states $N_{\text {2qp }} \geqslant 67,130$. In the present 3D calculation, even though the size of the space is reduced by incorporating the parity and the $z$-signature symmetry, the number of two-quasiparticle states easily exceeds 100 000. It requires enormous computational power and memory capacity to explicitly construct such large QRPA matrices. The FAM significantly reduces the computational burden and provides a feasible numerical approach to the QRPA.

Conclusions. Toward fully microscopic and nonempirical construction of the five-dimensional quadrupole collective Hamiltonian, we have developed a 3D FAM-QRPA code applicable to triaxially deformed nuclei with superfluidity. We demonstrated that the results showed good agreement with the previous axial QRPA results on multipole modes of excitation in the axially symmetric nuclei ${ }^{24} \mathrm{Mg}$ and ${ }^{100} \mathrm{Zr}$. In axially deformed nuclei, the quadrupole strength functions with a given $K$ value and different $r_{x}$ quantum numbers coincide with each other. The rotational zero-energy modes around the $x$ and $y$ axes exist, but those around the $z$ (symmetry) axis do not.

We applied our 3D FAM-QRPA to isoscalar quadrupole modes in the triaxially deformed superfluid nuclei ${ }^{110} \mathrm{Ru}$ and
${ }^{190} \mathrm{Pt}$. Five different peaks in the strength functions appear depending on $K$ and the signature $r_{x}$. Three rotational modes also emerge at zero energy, associated with rotations about all three axes $(x, y, z)$ because of the triaxial deformation.

The present FAM computation depends mainly on the numbers of the mesh points and of the HF-basis states. The computation of the isoscalar quadrupole strength for $201 \mathrm{\omega}$ points in Fig. 4(b) is about $350 \mathrm{CPU} h$ in total and 3.5-GB memory. This indicates the efficiency of our computational method and feasibility in currently available computational resources.

We intend to develop a parallelized local QRPA computer code based on the present FAM-QRPA framework to derive the collective inertial functions at every $(\beta, \gamma)$ point. To obtain low-lying discrete normal modes in the local QRPA, the contour integration technique of Ref. [38] may be useful. The extension of the present FAM-QRPA to the local QRPA is in progress.

Acknowledgments. The authors acknowledge N. Hinohara for fruitful discussions. This work was funded by ImPACT Program of Council for Science, Technology and Innovation (Cabinet Office, Government of Japan). Numerical calculations were performed, in part, using the COMA (PACS-IX) at the Center for Computational Sciences, University of Tsukuba.
[1] K. Heyde and J. L. Wood, Rev. Mod. Phys. 83, 1467 (2011).

[2] http://www.jst.go.jp/impact/en/program/08.html.

[3] M. Bender, P.-H. Heenen, and P.-G. Reinhard, Rev. Mod. Phys. 75, 121 (2003).

[4] M. Bender and P.-H. Heenen, Phys. Rev. C 78, 024309 (2008).

[5] T. R. Rodríguez and J. L. Egido, Phys. Rev. C 81, 064323 (2010).

[6] J. M. Yao, J. Meng, P. Ring, and D. Vretenar, Phys. Rev. C 81, 044311 (2010).

[7] M. Anguiano, J. L. Egido, and L. M. Robledo, Nucl. Phys. A 696, 467 (2001).

[8] J. Dobaczewski, M. V. Stoitsov, W. Nazarewicz, and P.-G. Reinhard, Phys. Rev. C 76, 054315 (2007).

[9] L. Próchniak, P. Quentin, D. Samsoen, and J. Libert, Nucl. Phys. A 730, 59 (2004).

[10] T. Nikšić, Z. P. Li, D. Vretenar, L. Próchniak, J. Meng, and P. Ring, Phys. Rev. C 79, 034303 (2009).

[11] J. P. Delaroche, M. Girod, J. Libert, H. Goutte, S. Hilaire, S. Péru, N. Pillet, and G. F. Bertsch, Phys. Rev. C 81, 014303 (2010).

[12] M. Matsuo, T. Nakatsukasa, and K. Matsuyanagi, Prog. Theor. Phys. 103, 959 (2000).

[13] T. Nakatsukasa, Prog. Theor. Exp. Phys. 2012, 01A207 (2012).

[14] T. Nakatsukasa, K. Matsuyanagi, M. Matsuo, and K. Yabana, Rev. Mod. Phys. 88, 045004 (2016).

[15] N. Hinohara, K. Sato, T. Nakatsukasa, M. Matsuo, and K. Matsuyanagi, Phys. Rev. C 82, 064313 (2010).

[16] N. Hinohara, K. Sato, K. Yoshida, T. Nakatsukasa, M. Matsuo, and K. Matsuyanagi, Phys. Rev. C 84, 061302(R) (2011).

[17] N. Hinohara, Z. P. Li, T. Nakatsukasa, T. Nikšić, and D. Vretenar, Phys. Rev. C 85, 024323 (2012).

[18] K. Yoshida and N. Hinohara, Phys. Rev. C 83, 061302(R) (2011).

[19] K. Yoshida and N. V. Giai, Phys. Rev. C 78, 064316 (2008).

[20] S. Péru and H. Goutte, Phys. Rev. C 77, 044313 (2008).
[21] D. Peña Arteaga, E. Khan, and P. Ring, Phys. Rev. C 79, 034311 (2009).

[22] C. Losa, A. Pastore, T. Døssing, E. Vigezzi, and R. A. Broglia, Phys. Rev. C 81, 064307 (2010).

[23] J. Terasaki and J. Engel, Phys. Rev. C 82, 034326 (2010).

[24] H. Imagawa and Y. Hashimoto, Phys. Rev. C 67, 037302 (2003).

[25] T. Inakura, T. Nakatsukasa, and K. Yabana, Phys. Rev. C 80, 044301 (2009).

[26] T. Inakura, T. Nakatsukasa, and K. Yabana, Phys. Rev. C 84, 021302(R) (2011).

[27] T. Inakura, T. Nakatsukasa, and K. Yabana, Phys. Rev. C 88, 051305(R) (2013).

[28] I. Stetcu, A. Bulgac, P. Magierski, and K. J. Roche, Phys. Rev. C 84, 051309 (2011).

[29] S. Ebata, T. Nakatsukasa, T. Inakura, K. Yoshida, Y. Hashimoto, and K. Yabana, Phys. Rev. C 82, 034306 (2010).

[30] G. Scamps and D. Lacroix, Phys. Rev. C 88, 044310 (2013).

[31] G. Scamps and D. Lacroix, Phys. Rev. C 89, 034314 (2014).

[32] S. Ebata, T. Nakatsukasa, and T. Inakura, Phys. Rev. C 90, 024303 (2014).

[33] T. Nakatsukasa, T. Inakura, and K. Yabana, Phys. Rev. C 76, 024318 (2007).

[34] P. Avogadro and T. Nakatsukasa, Phys. Rev. C 84, 014314 (2011).

[35] M. Stoitsov, M. Kortelainen, T. Nakatsukasa, C. Losa, and W. Nazarewicz, Phys. Rev. C 84, 041305(R) (2011).

[36] H. Liang, T. Nakatsukasa, Z. Niu, and J. Meng, Phys. Rev. C 87, 054310 (2013).

[37] P. Avogadro and T. Nakatsukasa, Phys. Rev. C 87, 014331 (2013)

[38] N. Hinohara, M. Kortelainen, and W. Nazarewicz, Phys. Rev. C 87, 064309 (2013). 
[39] T. Nikšić, N. Kralj, T. Tutiš, D. Vretenar, and P. Ring, Phys. Rev. C 88, 044327 (2013).

[40] M. T. Mustonen, T. Shafer, Z. Zenginerler, and J. Engel, Phys. Rev. C 90, 024308 (2014).

[41] J. C. Pei, M. Kortelainen, Y. N. Zhang, and F. R. Xu, Phys. Rev. C 90, 051304(R) (2014).

[42] N. Hinohara, M. Kortelainen, W. Nazarewicz, and E. Olsen, Phys. Rev. C 91, 044323 (2015).

[43] N. Hinohara, Phys. Rev. C 92, 034321 (2015).

[44] M. Kortelainen, N. Hinohara, and W. Nazarewicz, Phys. Rev. C 92, 051302(R) (2015).

[45] M. T. Mustonen and J. Engel, Phys. Rev. C 93, 014304 (2016).

[46] T. Oishi, M. Kortelainen, and N. Hinohara, Phys. Rev. C 93, 034329 (2016).

[47] N. Hinohara and W. Nazarewicz, Phys. Rev. Lett. 116, 152502 (2016).

[48] K. Wen and T. Nakatsukasa, Phys. Rev. C 94, 054618 (2016).

[49] K. Wen and T. Nakatsukasa, Phys. Rev. C 96, 014610 (2017).

[50] X. Sun and D. Lu, Phys. Rev. C 96, 024614 (2017).

[51] K. Wang, M. Kortelainen, and J. C. Pei, Phys. Rev. C 96, 031301(R) (2017).
[52] A. Baran, A. Bulgac, M. McNeil Forbes, G. Hagen, W. Nazarewicz, N. Schunck, and M. V. Stoitsov, Phys. Rev. C 78, 014318 (2008).

[53] P. Bonche, H. Flocard, and P.-H. Heenen, Nucl. Phys. A 467, 115 (1987).

[54] B. Gall, P. Bonche, J. Dobaczewski, H. Flocard, and P.-H. Heenen, Z. Phys. A: Hadrons Nucl. 348, 183 (1994).

[55] J. Terasaki, P.-H. Heenen, P. Bonche, J. Dobaczewski, and H. Flocard, Nucl. Phys. A 593, 1 (1995).

[56] P. Bonche, H. Flocard, and P.-H. Heenen, Comput. Phys. Commun. 171, 49 (2005).

[57] W. Ryssens, V. Hellemans, M. Bender, and P.-H. Heenen, Comput. Phys. Commun. 187, 175 (2015).

[58] P. Bonche, H. Flocard, P.-H. Heenen, S. Krieger, and M. Weiss, Nucl. Phys. A 443, 39 (1985).

[59] V. Hellemans, P.-H. Heenen, and M. Bender, Phys. Rev. C 85, 014326 (2012).

[60] J. Bartel, P. Quentin, M. Brack, C. Guet, and H.-B. Håkansson, Nucl. Phys. A 386, 79 (1982).

[61] E. Chabanat, P. Bonche, P. Haensel, J. Meyer, and R. Schaeffer, Nucl. Phys. A 635, 231 (1998).

[62] N. Hinohara (private communication).

[63] K. Yoshida, Phys. Rev. C 82, 034324 (2010). 